\title{
A Novel Channel Assignment Approach for Local Broadcast in Multi-radio Multi-channel Wireless Networks
}

\author{
Kai Xing Xiuzhen Cheng \\ Department of Computer Science \\ The George Washington University \\ Washington, DC 20052, USA \\ $\{$ kaix, cheng\}@gwu.edu
}

\begin{abstract}
This paper presents a novel channel assignment approach to support local broadcast in multi-radio multi-channel (MR-MC) wireless networks. The proposed algorithm intelligently assigns channels to each node based on superimposed codes. By applying superimposed codes, each node can easily locate a common channel shared with its $s$-closest neighbors. Under certain conditions, it is proved that our solution is able to solve the exposed terminal problem as well as the hidden terminal problem. Furthermore, with our channel assignment for local broadcast, most traditional MAC/routing protocols, though designed for single-radio single-channel (SR-SC) wireless networks, can possibly adapt to MR-MC wireless networks.
\end{abstract}

Keywords: multi-radio multi-channel wireless networks, local broadcast, channel assignment

\section{INTRODUCTION}

With recent advancements in wireless technology, the utilization of multiple radios and multiple channels provides an opportunity to greatly improve network capacity. In multi-radio multi-channel (MR-MC) wireless networks, nodes can communicate with multiple neighboring nodes simultaneously over different channels, and thus the network performance can be significantly improved through concurrent transmissions.

However, MR-MC wireless networks also bring in a challenge in supporting local broadcast. As defined in [1], local broadcast provides the capability for a packet transmitted by a node potentially to be received by all neighbors. It plays an important role in wireless communication, especially in the scenarios of efficiently disseminating information in the network, i.e., beaconing, broadcasting, efficient routing, etc. Unfortunately,

$1-4244-1513-06 / 07 / \$ 25.00$ (c) 2007 IEEE since neighboring pairs of nodes tend to use different channels in MR-MC wireless networks, neighborhood communications may no longer share a common channel, thereby preclude local broadcast and lead to network topology changes and inefficient communications.

Therefore, a key challenge in MR-MC wireless networks is to provide effective support for local broadcast, in order to improve communication efficiency. For example, if all nodes in the network fix an interface on some common channel, local broadcast can be easily achieved over that channel. But interference is produced among neighboring nodes and other channel resources are wasted. On the other hand, if nodes tune into different channels, some neighboring nodes may not share any common channel, thereby reduce the efficiency of beaconing/local broadcast and result in extra transmissions in neighborhood communication.

In supporting local broadcast in MR-MC wireless networks, a node should share at least one common channel with its neighbors, and two neighbors should utilize different channels for local broadcast if their neighborhood is different. However, current channel coordination/assignment approaches lack a support of local broadcast in MR-MC wireless networks: as neighboring nodes tend to use different channels for transmission, the sender has to separately transmit a packet on multiple channels to achieve local broadcast. Thus, the cost of local broadcast in MR-MC networks can be more expensive than that in single-radio single-channel (SR-SC) networks.

Additionally, since the dynamic channel coordination/assignment may result in topology changes, it may break neighborhood connectivity and further lead to inefficient routing or even network partition. This pitfall renders most traditional wireless MAC/routing protocols inapplicable in MR-MC wireless networks, since most of 
these protocols assume that a node can always communicate with its neighbors within its transmission range.

In this paper we propose a novel channel assignment algorithm aiming at providing effective support of local broadcast in MR-MC wireless networks. If the neighborhood of each node in the network is different, this algorithm intelligently assigns a channel to each node for local broadcast. To be specific, our algorithm first computes a channel assignment matrix, with each column representing a channel assignment codeword. Then each node randomly picks a unique column from the matrix. With simple binary AND/OR operations on the codewords obtained from neighboring nodes, each node can locate a common channel shared with its onehop neighbors. Based on our analysis, this algorithm can solve the exposed terminal problem and the hidden terminal problem under certain conditions. Simulation study indicates that our algorithm can greatly alleviate communication interference without introducing complex coordination/scheduling.

The rest of the paper is organized as follows: Section II discusses the channel coordination/assignment techniques in MR-MC wireless networks and introduces the background knowledge on superimposed disjunct code. In Section III, we present the network model. Section IV details our channel assignment algorithm. Section V studies its properties and performance on interference and Section VI provides a short discussion on its additional properties. Section VII summarizes our work and concludes the paper.

\section{BACKGROUND KNOWLEDGE}

\section{A. Channel Coordination in MR-MC networks}

Local broadcast has been widely used in various wireless communication scenarios, i.e., beaconing, broadcasting, and routing, to efficiently disseminate information in the network. The approach used to support local broadcast in MR-MC wireless networks depends on the techniques in use for channel coordination, which can be classified into two categories: static and dynamic.

Static approaches [2] [3] coordinate nodes on a fixed channel. It needs simple coordination, but wastes the multi-channel resources and may lead to undesirable interference during beaconing/local broadcast. Dynamic channel coordination allows each node dynamically meet with others on different channels [4] [5]. It makes good use of channel resources, but requires complex scheduling/channel coordination as well as synchronization. Furthermore, channel switch may incur extra delay, overhead, and topology changes, sometimes even leads to network partition.
For example, when channels are coordinated through periodic rendezvous/pseudorandom sequence on a common channel [4] [5], local broadcast can be done during the rendezvous interval. Compared with SR-SC networks, this technique does not increase the local broadcast cost, but increases the transmission delay, requires tight clock synchronization, and produces extra overhead during channel switch. In techniques where nodes use different fixed channels, a node may have to transmit the local broadcast packet multiple times over different channels, which incurs higher overhead than that in SRSC wireless networks.

A hybrid Dynamic Channel Assignment approach (DCA) is proposed in [6]. In DCA, there are one control channel and multiple data channels. One radio stays on the control channel for exchanging control messages to coordinate data channels, and the other radios dynamically switch to one of the data channels for transmission. A similar technique is adopted by Krishna et al. [7] to design a centralized interference-aware hybrid channel coordination algorithm. The proposed algorithm forces one radio on each node to operate on a default channel. Based on multi-radio conflict graph built over the estimated interference, the Breadth-First-Search channel assignment server assigns channels to other radios. Another hybrid approach with loose synchronization is proposed in [8]. In this approach, each node fixes one radio on some channel (different nodes possibly use different fixed channels), while the remaining radios of the node dynamically switch to the fixed channels of its neighboring nodes.

However, most approaches mentioned above are designed for unicast. They require either complex scheduling/coordination, or extra transmissions over different channels for local broadcast, both leading to undesired delay, overheads or inefficiency. To our best knowledge, the most related work is proposed in [9], which provides heuristic approaches to decrease broadcast latency in MR-MC wireless mesh networks. In this paper, we propose a novel method to realize local broadcast effectively and efficiently based on superimposed $s$-disjunct codes.

\section{B. s-disjunct codes}

Given two binary codewords (vectors) $y$ and $z$ with the same length, we say that $y$ covers $z$ if the Boolean sum $y \bigvee z=y$. A binary code can be represented as a binary matrix, with each column corresponding to a codeword.

Superimposed code (SC): A $N \times t$ binary matrix $X$ is called a superimposed code of length $N$, size $t$, strength 


$$
\left(\begin{array}{lllllllllllll}
1 & 0 & 0 & 0 & 1 & 0 & 0 & 0 & 0 & 0 & 1 & 0 & 1 \\
1 & 1 & 0 & 0 & 0 & 1 & 0 & 0 & 0 & 0 & 0 & 1 & 0 \\
0 & 1 & 1 & 0 & 0 & 0 & 1 & 0 & 0 & 0 & 0 & 0 & 1 \\
1 & 0 & 1 & 1 & 0 & 0 & 0 & 1 & 0 & 0 & 0 & 0 & 0 \\
0 & 1 & 0 & 1 & 1 & 0 & 0 & 0 & 1 & 0 & 0 & 0 & 0 \\
0 & 0 & 1 & 0 & 1 & 1 & 0 & 0 & 0 & 1 & 0 & 0 & 0 \\
0 & 0 & 0 & 1 & 0 & 1 & 1 & 0 & 0 & 0 & 1 & 0 & 0 \\
0 & 0 & 0 & 0 & 1 & 0 & 1 & 1 & 0 & 0 & 0 & 1 & 0 \\
0 & 0 & 0 & 0 & 0 & 1 & 0 & 1 & 1 & 0 & 0 & 0 & 1 \\
1 & 0 & 0 & 0 & 0 & 0 & 1 & 0 & 1 & 1 & 0 & 0 & 0 \\
0 & 1 & 0 & 0 & 0 & 0 & 0 & 1 & 0 & 1 & 1 & 0 & 0 \\
0 & 0 & 1 & 0 & 0 & 0 & 0 & 0 & 1 & 0 & 1 & 1 & 0 \\
0 & 0 & 0 & 1 & 0 & 0 & 0 & 0 & 0 & 1 & 0 & 1 & 1
\end{array}\right)
$$

Fig. 1. An example of a superimposed $(3,1,13)$-code of size 13

$s$, and listsize $\leq L-1$ if the Boolean sum of any $s$ subset of the codewords in $X$ can cover not more than $L-1$ codewords that are not components of the $s$-subset. This code is also called a $(s, L, N)$-code of size $t$. The matrix in Fig 1 shows an example of a superimposed $(3,1,13)$-code of size 13 .

$s$-disjunct Code: A binary matrix $X$ is called an $s$ disjunct code if and only if it has the property that the Boolean sum of any $s$ codewords in $X$ does not cover any codeword not in that set of $s$ codewords.

Based on the definitions above, a superimposed $(s, 1, N)$-code is an $s$-disjunct code. Taking the $(3,1,13)$-code shown in Fig. 1 as an example, the Boolean sum of the first 3 codewords of $X$ is $X(1) \bigvee X(2) \bigvee X(3)=$ $(1,1,1,1,1,1,0,0,0,1,1,1,0)^{\prime}$, which doesn't cover any other codeword of $X$ but the codewords themselves used in the computation of the boolean sum.

According to the $s$-disjunct characteristic of superimposed $s$-disjunct codes, we derive the following important property:

Property 2.1: Given a superimposed s-disjunct code $X$, let $z$ be any designated codeword in $X$. For any set of $s+1$ codewords containing $z$, there exists at least one row in $X$ that intersects $z$ with a value ' 1 ' and the other $s$ columns with a value ' 0 '

Note that methods of constructing a good superimposed $s$-disjunct code have been extensively studied [10] [11] [12] [13] [14] [15]. We will not discuss these construction methods in this paper.

\section{NeTwORK MOdEL}

We consider a static wireless network with nodes arbitrarily distributed on a plane. This network can be modelled with a directed graph $G(V, E)$, where $V$ is the set of nodes in the network, and $E$ is the set of directed links for communication. Given a node $u \in V$, let $\mathcal{N}(u)$ denote the set of neighboring nodes within node $u$ 's transmission range, $\mathcal{N}_{i}(u)$ denote the $i$-th closest neighboring node of $u, \mathcal{N}^{2}(u)$ denote the set of nodes that may interfere node $u$ 's transmission. Note that $\mathcal{N}(u)$ is a subset of $\mathcal{N}^{2}(u)$. When local broadcast is needed at node $u, u$ 's neighbors will tune one of their radios on $u$ 's local broadcast channel.

We also assume there exist $N$ orthogonal channels in the network. A local broadcast transmission on channel $k$, where $k \in 1, \cdots, N$, is successful if and only if all potential interferers in the neighborhood of the receivers are silent on channel $k$ during the transmission.

\section{A Channel Assignment Algorithm for LOCAL BROADCAST}

In this section, we describe our channel assignment algorithm to support local broadcast, based on the superimposed $s$-disjunct code.

We assume that for any node $u$ in the network, the node set $\{\mathcal{N}(u)\}$ is unique. Based on Property 2.1, we can construct a connection between $s$-disjunct codes and channel assignments to support local broadcast in MRMC wireless networks.

\section{A. The Channel Assignment Algorithm}

Given an MR-MC wireless network with $N$ available channels, we generate a superimposed $s$-disjunct code $X$ as the channel assignment matrix, where $X$ is a $N \times t$ binary matrix, with $N$ representing the number of channels, and $t$ representing the number of nodes in the network.

Each node $u$ in the network picks a unique codeword from $X$, denoted by $X(u)$, as its channel assignment codeword. Each element of $X(u)$ corresponds to a channel and its $1 / 0$ value represents that this channel can be used or not.

Let $X\left(\mathcal{N}^{2}(u)\right)$ denote the set of channel assignment codewords of the node set $\mathcal{N}^{2}(u)$, the set of interferers of node $u$. We propose our algorithm (Algorithm 1) to locate one channel for node $u$ 's local broadcast. The basic idea can be stated as follows: for $\forall u \in V$, node $u$ first identifies those channels not being used by any of its $s$-closest neighbors. According to Property 2.1 , node $u$ can always find such a channel. If there are more than one channel available, $u$ picks the 
one that is in least use among $u$ 's all other interferers: $\mathcal{N}_{s+1}(u), \cdots, \mathcal{N}_{\left|\mathcal{N}^{2}(u)\right|}(u)$.

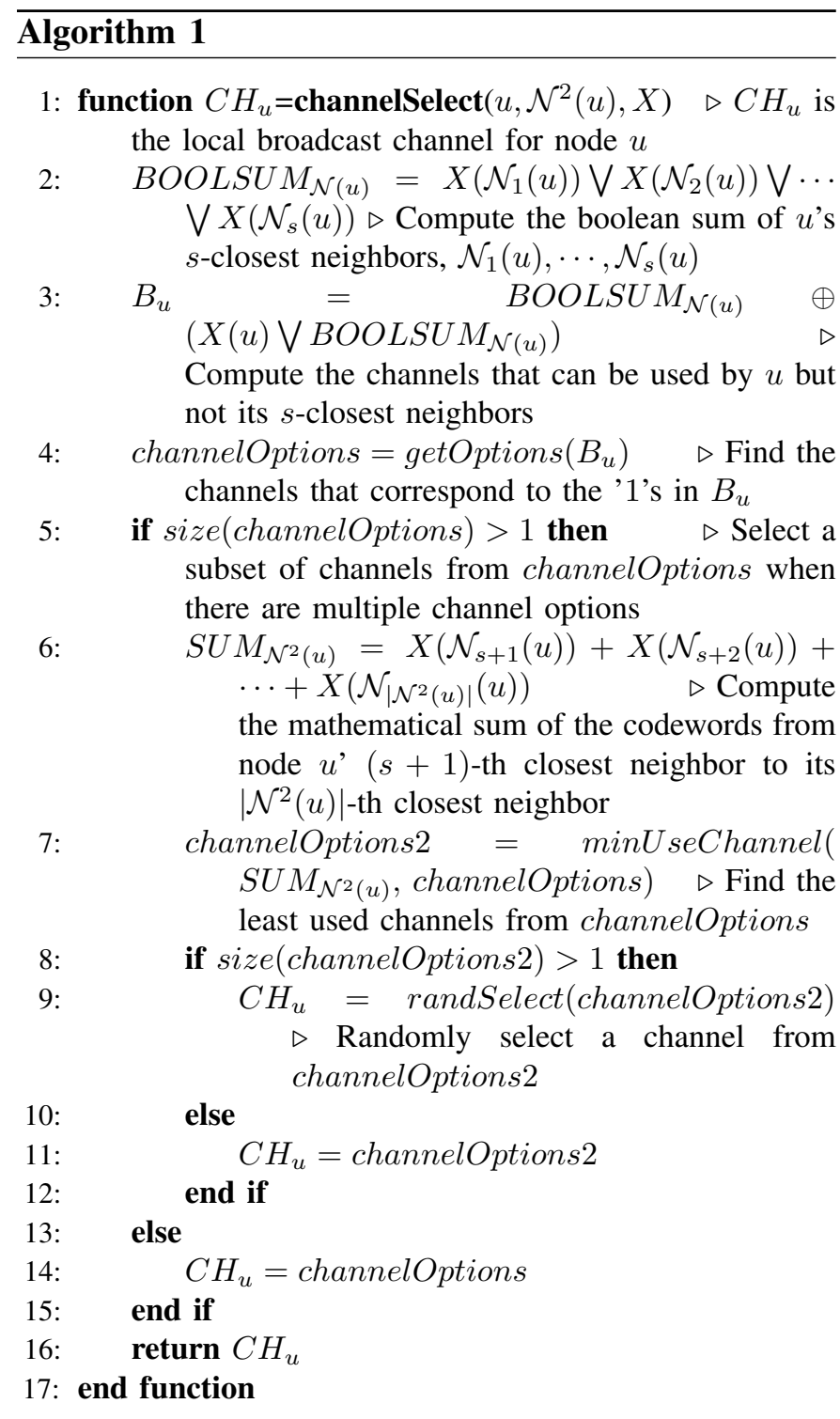

The inputs to Algorithm 1 include $X(u)$ and $X\left(\mathcal{N}^{2}(u)\right)$. We first compute the Boolean sum $B O O L S U M_{\mathcal{N}(u)}$ of $X\left(\mathcal{N}_{1}(u)\right), \cdots, X\left(\mathcal{N}_{s}(u)\right)$. Then $B_{u}$, a binary vector whose value ' 1 ' corresponds to the rows having a value ' 0 ' in $X\left(\mathcal{N}_{1}(u)\right), \cdots, X\left(\mathcal{N}_{s}(u)\right)$ and a value ' 1 ' in $X(u)$, is computed. These rows represent the channels not being used by any of the $s$ closest interferers, except $u$ itself. Node $u$ puts these channels into channelOptions. If channelOptions contains more than one channel, node $u$ selects the least used one among $u$ 's all other interferers', namely $\mathcal{N}_{s+1}(u), \cdots, \mathcal{N}_{\left|\mathcal{N}^{2}(u)\right|}(u)$.

Example: Take the superimposed 3-disjunct code $X$ in Fig 1 as an example. Given a node $u$, its 3 closest neighboring nodes $v, w, z$, and its two- hop neighbors $a, b, c, d$. Suppose $X(u)=X(1)$, $X(v)=X(2), X(w)=X(3), X(z)=X(4)$, $X(a)=X(5), X(b)=X(6), X(c)=X(7)$ and $X(d)=X(8)$, respectively. According to the algorithm, $B O O L S U M_{\mathcal{N}(u)}=(0,1,1,1,1,1,1,0,0,0,1,1,1)^{\prime}$, and $B_{u}=(1,0,0,0,0,0,0,0,0,1,0,0,0)^{\prime}$. Therefore channelOptions $=\{1,10\}$, and channelOptions $2=$ $\{1,10\}$. Then we randomly choose a channel from the channel set $\{1,10\}$ for node $u$ 's local broadcast.

According to Property 2.1, with the neighborhood channel assignment codewords, each node in the network can always find one channel for local broadcast, and the selected channel is guaranteed not to be used by its $s$ closest neighbors.

\section{B. Enhanced Neighborhood Connectivity Design}

Note that for a superimposed $s$-disjunct code of length $N$ and size $t$, the upper bound of $s$ is limited by $N$ [16] ( $s$ can be as large as $N-1$ when $N=t$ ). Therefore in our proposed algorithm, $s$, representing the neighborhood connectivity, cannot be a large number if the number of available channels $N$ in the network is small. However, this should not be a restriction on the application of our channel assignment algorithm in IEEE 802.16 based MR-MC wireless networks [17]. The OFDMA technique in IEEE 802.16e [18] [19] allows bandwidth to be divided into many lower-speed subchannels to increase resistance to multi-path interference. Typically a large number of non-overlapping orthogonal sub-channels may be available for simultaneous transmissions. Therefore, in this type of MR-MC wireless networks $s$ could be sufficiently large because of the large number of available non-overlapping orthogonal sub-channels.

Since the non-overlapping channels in 802.11 standards are limited (3 non-overlapping channels in IEEE $802.11 \mathrm{~b} / \mathrm{g} ; 12$ non-overlapping channels in the original IEEE 802.11a), $s$ in 802.11 wireless networks is limited to some small number, which may affect the network connectivity and lead to inefficient communication. Therefore, in order to apply the algorithm in such networks, the nodes in the network should be carefully deployed such that each node has a small number of neighbors while the network is still connected.

Note that a superimposed $s$-disjunct code guarantees success in the case of $s$ or less number of neighbors, but it is very likely that we still have disjunct property with more than $s$ codewords. Here we introduce the definition of $\alpha$-almost $s$-disjunct code proposed in [20] [21]: $A$ binary matrix is $\alpha$-almost $s$-disjunct if for any randomly selected set of s columns, the probability that they cover no other column is at least $\alpha$. 


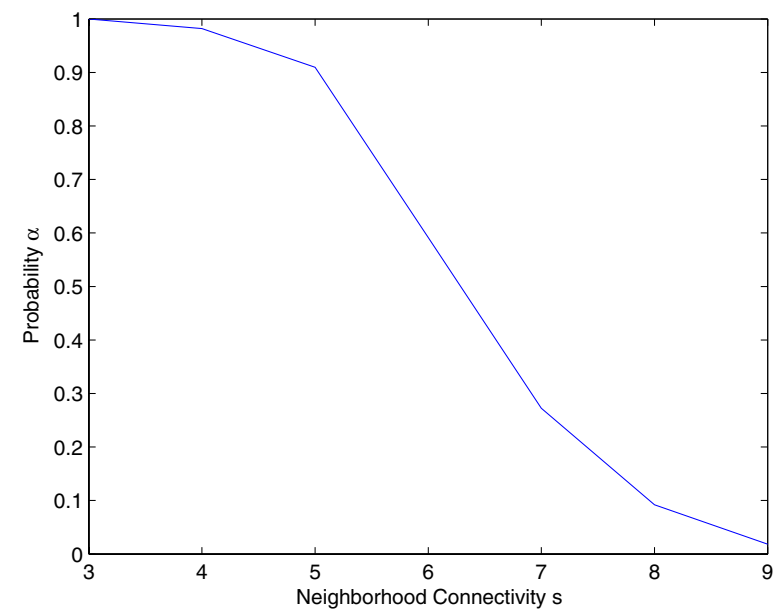

Fig. 2. Probability $\alpha$ vs. neighborhood connectivity $s$ based on a superimposed $(3,1,13)$-code.

Taking the superimposed $(3,1,13)$-code in Fig. 1 as an example. The superimposed $(3,1,13)$-code is 1almost 3-disjunct, 0.98-almost 4-disjunct, 0.91-almost 5disjunct, and 0.6-almost 6-disjunct, as shown in Fig. 2. This means that we can extend $s$ to a larger number. In other words, we first divide the channel assignment codewords into subsets where the codewords in each subset hold the disjunct property with a larger $s$. Then neighboring nodes pick channel assignment options (codewords) from a subset instead of the whole set.

\section{Performance Analysis}

In this section, we study the properties of our channel assignment algorithm for local broadcast. We conclude that our algorithm can solve the exposed terminal problem and the hidden terminal problem under certain conditions. The simulation study indicates that in the general case our algorithm significantly alleviate the communication interference in the network.

\section{A. Theoretical Study}

Theorem 5.1: Given two arbitrary nodes $u$ and $v$ adjacent to each other, if we have $|\mathcal{N}(u)| \leq s$ and $|\mathcal{N}(v)| \leq$ $s$, and if $\{\mathcal{N}(u)\} \backslash\{\mathcal{N}(v)\} \neq\{\mathcal{N}(v)\} \backslash\{\mathcal{N}(u)\}$, the proposed channel assignment algorithm can guarantee that the local broadcasts of the two nodes are free of interference from each other.

Proof. Let $X(u)$ and $X(v)$ denote the channel assignment codewords of node $u$ and node $v$ respectively. Based on the $s+1$ channel assignment codewords obtained from node $u$ and its $s$ nearest neighboring nodes, according to Property 2.1 , node $u$ can locate a channel for local broadcast. The row in $X$ corresponding to the local broadcast channel intersects $X(u)$ with a value ' 1 ' and the other $s$ columns of $\mathcal{N}(u)$ with a value ' 0 '. Let $X_{k}$ denote this row.

Since node $v$ is a neighboring node of node $u$, it is easily to see that the value of $X(v)$ at the row $X_{k}$ is 0 , which means that the channel corresponding to the row $X_{k}$ is never assigned to node $v$ for local broadcast.

Same argument holds for node $v$.

Therefore, every pair of neighboring nodes $u$ and $v$ use different channels for local broadcast. They cause no interference between each other.

The hidden/exposed terminal problems are wellknown phenomenons in wireless networks due to the broadcast nature of the wireless medium. For example, in Fig. 3(a), when node $A$ is transmitting data to node $B$, the hidden terminal problem occurs when node $C$, which is unaware of the ongoing transmission, attempts to transmit, thus causing collision at node $B$; in Fig.3(b), when node $B$ is transmitting data to node $A$, the exposed terminal problem occurs when node $C$, which is aware of the ongoing transmission, refrains to communicate with $D$, thus causing degraded network throughput.

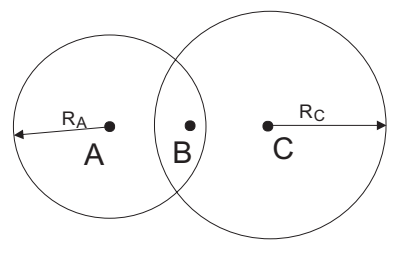

(a)

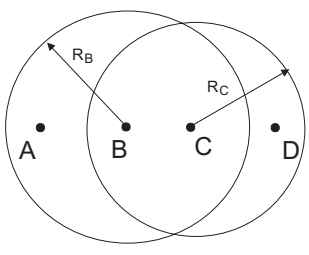

(b)
Fig. 3. Hidden and exposed terminal problems in wireless networks.

With the proposed algorithms in Section IV, we can prove that the exposed/hidden terminal problems during local broadcast can be solved under certain conditions.

Theorem 5.2: If $\forall u \in V,|\mathcal{N}(u)| \leq s$ holds, with the proposed channel assignment algorithm, the exposed terminal problem during local broadcast in MR-MC wireless networks can be solved.

Proof. Given any two nodes $B$ and $C$ that can potentially result in the exposed terminal problem, as shown in Fig. 3(b). According to Theorem 5.1, since node $B$ and node $C$ are neighbors, they use different channels and are free of interference for local broadcast.

Therefore there is no interference during local broadcast among neighboring nodes in the network. The exposed terminal problem can be solved in the network. 
Theorem 5.3: If $\left|\mathcal{N}^{2}(u)\right| \leq s$ holds for $\forall u \in V$, with the proposed channel assignment algorithm, the hidden terminal problem during local broadcast in MRMC wireless networks can be solved.

Proof. Given any two nodes $A$ and $C$ that can potentially result in the hidden terminal problem, as shown in Fig.3(a). According to Algorithm 1, node $A$ selects a channel whose corresponding row has a value ' 1 ' in $X(A)$ and a value ' 0 ' in $X(C)$. Namely the channel selected by node $A$ cannot be used by node $C$. According to Property 2.1 , node $A$ can always find such a channel for local broadcast.

The same argument holds for node $C$. Hence, node $A$ and $C$ use different channels and are free of interference during local broadcast.

Therefore, there is no interference during local broadcast in the network. The hidden terminal problem can be solved in the network.

\section{B. Simulation Study}

To evaluate the performance of our algorithm, we conduct a comparative study between our algorithm and the random channel assignment scheme (in which each node randomly chooses a channel for the local broadcast) via simulation.

In the simulation we have considered an area of $100 \times 100$ square unit with 13 randomly deployed nodes . The average node degree is set to 3 . We assume that the number of local broadcasts generated in the network during one second follows the Poisson distribution with a mean $\lambda$ (the local broadcast generating rate). The simulation setup is as follows:

- The total simulation time is 500 seconds;

- All simulation results are averaged over 20 different static topologies;

- Under each topology, the transmission range of each node is fixed such that the average node degree in the network is 3 ;

- The number of available channels in the network is set to 13 ;

- The local broadcast generating rate $\lambda$ is chosen from $3,4, \cdots, 9$ for each simulation.

During the simulation, in order to track and evaluate the interference in the network, we use the number of nodes that are interfered per second as our performance metric. Fig 4 shows that our algorithm has much fewer nodes interfered in the network than that of the random channel assignment scheme. It is also interesting to observe that when $\lambda$ increases, the number of nodes interfered increases at a slower speed in our algorithm than that in the random channel assignment scheme.

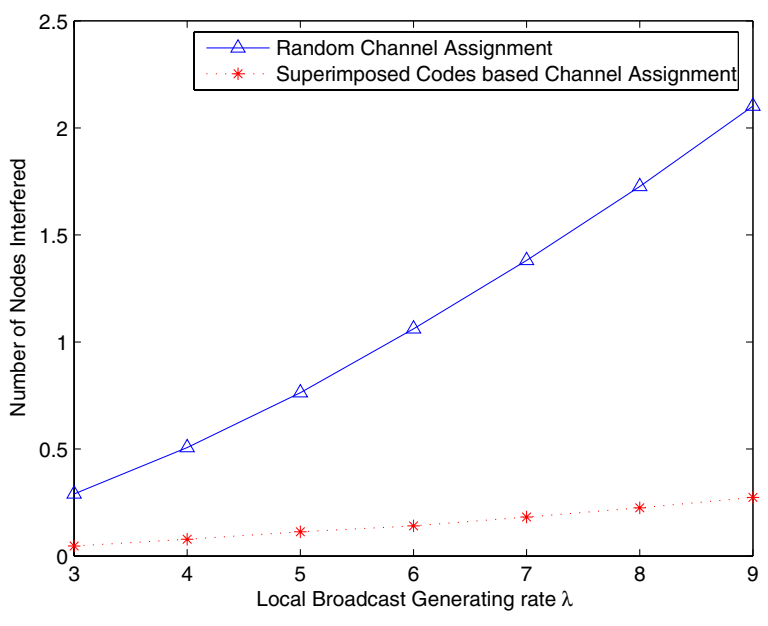

Fig. 4. The number of nodes interfered vs. the local broadcast generating rate $\lambda$.

From the analysis and the simulation results shown above, we conclude that based on our channel assignment scheme, the exposed/hidden terminal problems can be relieved because every pair of one-hop neighboring nodes (two-hop neighboring nodes) tend to use different channels for local broadcast. The interference can be reduced by at least $80 \%$ in our algorithm compared with that in the random channel assignment scheme, as shown in Fig.4.

\section{DISCUSSION}

Fairness is another important issue in channel utilization in wireless networks. Since the number of ' 1 's in each column of $X$ is a constant, each node in the network is assigned with the same number of channels in its channel assignment codeword, which guarantees fairness.

There is an important property of our channel assignment algorithm: the channels selected for local broadcast can be considered as the common channels shared by neighboring nodes. This leads to a major difference of our algorithm and current channel assignment approaches in MR-MC wireless mesh networks.

In current channel assignment approaches in MR$\mathrm{MC}$ wireless networks, most of them are designed for unicast, therefore lacking the support of local broadcast. As indicated in [1] [9], a channel assignment that performs well for unicast does not necessarily support local broadcast. It is possible that two nodes close to each other in distance but cannot communicate with each other because they do not share a common channel. Sometimes a node can communicate with the nodes far from it, but cannot communicate with the nodes close 
to it. Our algorithm can be considered as a complement solution to those protocols for local broadcast.

Note that most traditional MAC and routing protocols in SR-SC wireless networks are designed based on the following distance criteria: if nodes are within each other's transmission range, they can communicate with each other. Because the distance criteria is not well supported in current channel assignment approaches in MRMC wireless networks, the applicability of traditional MAC and routing protocols is greatly limited in MRMC wireless networks.

However, if $|\mathcal{N}(u)| \leq s$ holds for $\forall u \in V$ in the network, with the proposed algorithm, traditional MAC and routing protocols can easily be adapted to MRMC wireless networks, because our algorithm sustains the distance criteria: for neighboring nodes within each other's transmission range, they share a common channel for communication

\section{CONCLUSION}

In this paper, we have proposed a novel channel assignment algorithm based on the superimposed $s$ disjunct codes to efficiently support local broadcast in multi-radio multi-channel wireless networks. In our analysis and simulation study, it is shown that this algorithm has the ability to relieve the exposed/hidden terminal problems and provides local broadcast with much fewer collisions/interferences than the random channel assignment scheme. In addition, our algorithm provides a practical way that makes traditional wireless MAC/routing protocols adaptable in MR-MC wireless networks. As a future research, we plan to further evaluate this algorithm on testbeds of MR-MC wireless networks.

\section{REFERENCES}

[1] P. Kyasanur, J. So, C. Chereddi, and N. H. Vaidya, "Multichannel mesh networks: challenges and protocols," in IEEE Wireless Communications, vol. 13, no. 2, 2006, pp. 30-36.

[2] R. Draves, J. Padhye, and B. Zill, "Routing in multi-radio, multi-hop wireless mesh networks," in MobiCom '04: Proceedings of the 10th annual international conference on Mobile computing and networking, 2004, pp. 114-128.

[3] A. Raniwala, K. Gopalan, and T. cker Chiueh, "Centralized channel assignment and routing algorithms for multi-channel wireless mesh networks," SIGMOBILE Mob. Comput. Commun. Rev., vol. 8, no. 2, pp. 50-65, 2004.

[4] J. So and N. H. Vaidya, "Multi-channel mac for ad hoc networks: handling multi-channel hidden terminals using a single transceiver," in MobiHoc '04: Proceedings of the 5th ACM international symposium on Mobile ad hoc networking and computing. New York, NY, USA: ACM Press, 2004, pp. 222233.

[5] P. Bahl, R. Chandra, and J. Dunagan, "Ssch: slotted seeded channel hopping for capacity improvement in ieee 802.11 adhoc wireless networks," in MobiCom '04: Proceedings of the 10th annual international conference on Mobile computing and networking, 2004, pp. 216-230.
[6] S.-L. Wu, C.-Y. Lin, Y.-C. Tseng, and J.-P. Sheu, "A new multichannel mac protocol with on-demand channel assignment for multi-hop mobile ad hoc networks," in ISPAN '00: Proceedings of the 2000 International Symposium on Parallel Architectures, Algorithms and Networks (ISPAN '00). Washington, DC, USA: IEEE Computer Society, 2000, p. 232.

[7] K. N. Ramachandran, E. M. Belding, K. C. Almeroth, and M. M. Buddhikot, "Interference-aware channel assignment in multi-radio wireless mesh networks," in Infocom, 2006.

[8] P.Kyasanur and N.H.Vaidya, "Routing and interface assignment in multi-channel multi-interface wireless networks," in Wireless Communications and Networking Conference, 2005 IEEE, vol. 4, 2005, pp. 2051-2056.

[9] J. Qadir, C. T. Chou, and A. Misra, "Minimum latency broadcasting in multi-radio multi-channel multi-rate wireless mesh networks," in Technical Report, 2006.

[10] A. E. F. Clementi, A. Monti, and R. Silvestri, "Selective families, superimposed codes, and broadcasting on unknown radio networks," in SODA '01: Proceedings of the twelfth annual ACM-SIAM symposium on Discrete algorithms. Philadelphia, PA, USA: Society for Industrial and Applied Mathematics, 2001, pp. 709-718.

[11] A. D. Bonis and U. Vaccaro, "Constructions of generalized superimposed codes with applications to group testing and conflict resolution in multiple access channels," in Theoretical Computer Science 306, vol. 1, no. 3, 2003, pp. 223-243.

[12] A. D'yachkov, V. Lebedev, P. Vilenkin, and S. Yekhanin, "Cover-free families and superimposed codes: Constructions, bounds, and applications to cryptography and group testing," in IEEE International Symposium on Information Theory, 2001.

[13] D. Danev, "Some constructions of superimposed codes in euclidean spaces," Discrete Appl. Math., vol. 128, no. 1, pp. 85-101, 2003.

[14] A. D. yachkov, A. M. Jr, and V.V.Rykov, "New constructions of superimposed codes," in Information Theory, IEEE Transactions on, vol. 46, no. 1, 2000, pp. 284-290.

[15] H.-L. Fu and F. Hwang, "A novel use of t-packings to construct d-disjunct matrices," in Discrete Applied Mathematics, vol. 154, no. 12,2006 , pp. 1759-1762.

[16] A. G. D'yachkov and V. V. Rykov, "Optimal superimposed codes and designs for renyi's search model," Journal of Statistical Planning and Inference, vol. 100, no. 2, pp. 281-302, 2002.

[17] H.-Y. Wei, S. Ganguly, R.Izmailov, and Z.J.Haas, "Interferenceaware ieee 802.16 wimax mesh networks," Vehicular Technology Conference, 2005. VTC 2005-Spring. 2005 IEEE 61st, vol. 5, pp. 3102-3106, 2005.

[18] "Wimax/802.16 revealed." [Online]. Available: http://www.wifiplanet.com/tutorials/article.php/3550476

[19] "What is ieee 802.16e." [Online]. Available: http://www.wimax.com/education/faq/faq45

[20] D. C. Engelhart and A. Sivasubramaniam, "Almost disjunct codes in large scale multihop wireless network media access control,' in MASCOTS '05: Proceedings of the 13th IEEE International Symposium on Modeling, Analysis, and Simulation of Computer and Telecommunication Systems. Washington, DC, USA: IEEE Computer Society, 2005, pp. 453-463.

[21] A. J. Macula, V. V. Rykov, and S. Yekhanin, "Trivial two-stage group testing for complexes using almost disjunct matrices," Discrete and Applied Mathematics, vol. 137, pp. 97-107, 2004. 